\title{
Information and Announcement on the First Global Patient Safety Challenge: Clean Care is Safer Care
}

\author{
Julie Storr $^{1}$, Benedetta Allegranzi ${ }^{1}$, Agnes Leotsakos ${ }^{2}$, Didier Pittet ${ }^{1}$ \\ ${ }^{1}$ First Global Patient Safety Challenge, WHO World Alliance for Patient Safety, \\ ${ }^{2}$ Partnerships and Advocacy, WHO World Alliance for Patient Safety
}

Available from: http://www.ijic.info

\section{Background:}

In May 2002, the WHO World Health Assembly passed resolution WHA55.18 which urged countries to pay the greatest possible attention to patient safety. The resolution has ensured that the drive for safer health care is becoming a worldwide endeavor, bringing significant benefits to patients in all corners of the globe. In October 2004, WHO launched the World Alliance for Patient Safety (WAPS). The Alliance is concerned with raising awareness and political commitment in relation to improved patient safety. Each year, the Alliance delivers a number of programmes covering systemic and technical aspects to improve patient safety around the world.

\section{Introduction: Clean Care is Safer Care}

An essential element of the Alliance is the periodic identification of a theme covering an issue of universal relevance to all Member States. The first Global Patient Safety Challenge is one of the flagship programmes of the WAPS, concerned with galvanizing global commitment and action on the reduction of health careassociated infection (HAI). This is to be achieved through the promotion of clean, safe health care. Its main output has been a WHO Guideline, the first of its kind to address hand hygiene in the context of health care ${ }^{1}$. Together with the most comprehensive review of available evidence, the Guidelines and associated implementation tools, have formed a central tenet of the work of the First Global Patient Safety Challenge. In particular, emerging from the technical work has been an innovative concept
(My Five Moments for Hand Hygiene ${ }^{2}$ ) designed to revolutionize training and understanding in relation to hand hygiene improvement.

An integrated approach has involved the promotion of clean safe care in its broadest sense, and the First Global Patient Safety Challenge supports exiting WHO programmes relating to blood safety, injection and immunization safety, safe water and sanitation and safe surgery. To put into practice the WHO Guideline recommendations, a multifaceted implementation strategy has been developed related to the nine recommendations contained within the WHO Guidelines on Hand Hygiene in Health Care (Advanced Draft) combined with a package of practical tools to facilitate implementation activities at facility level (figure 1). 


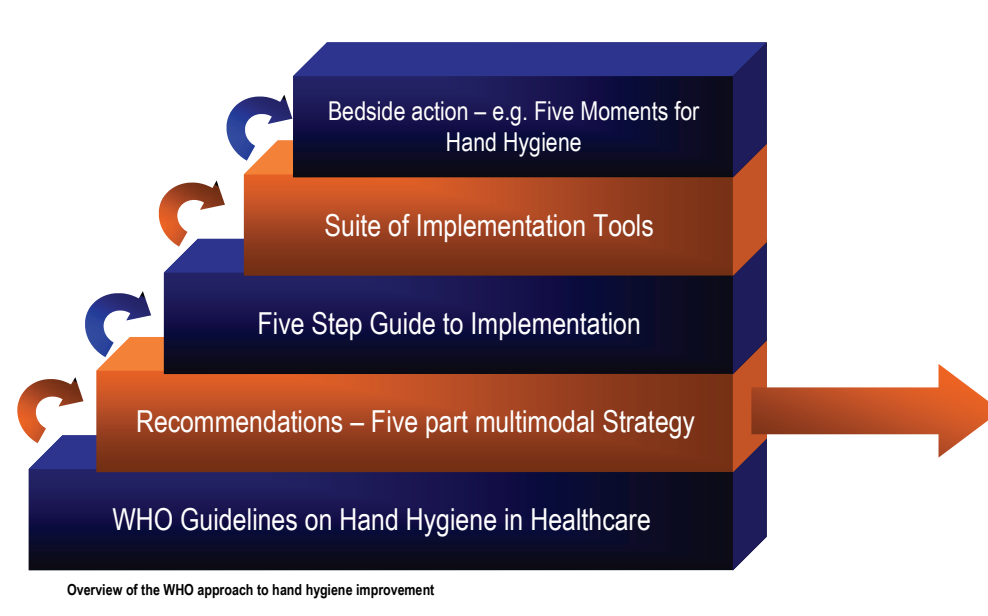

Figure 1: WHO practical tools to facilitate implementation activities at facility level

\section{Field Testing}

Implementation of the WHO Multimodal Strategy for hand hygiene improvement (based on the WHO Guidelines) is being pilot tested in each of the six WHO regions. The aim of the testing phase is to evaluate the feasibility and acceptability of the recommendations expressed in the guidelines, in a wide range of different healthcare settings and learn from the successes and shortcomings. Work is ongoing in Bangladesh, Costa Rica, Hong Kong SAR, Italy, Mali, Pakistan, Russia, and Saudi Arabia. Each test site implements the WHO Multimodal Strategy and follows a Five-Step implementation process (figure 2). In many areas of the world, access to

\section{Your 5 moments for} HAND HYGIENE

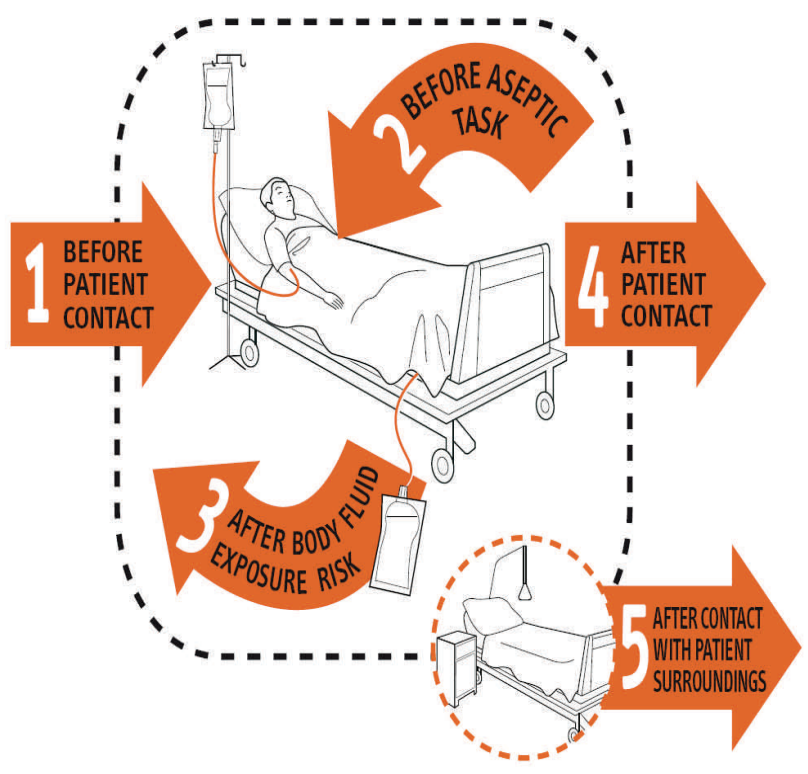

Figure 2: WHO Five Moments of Hand Hygiene

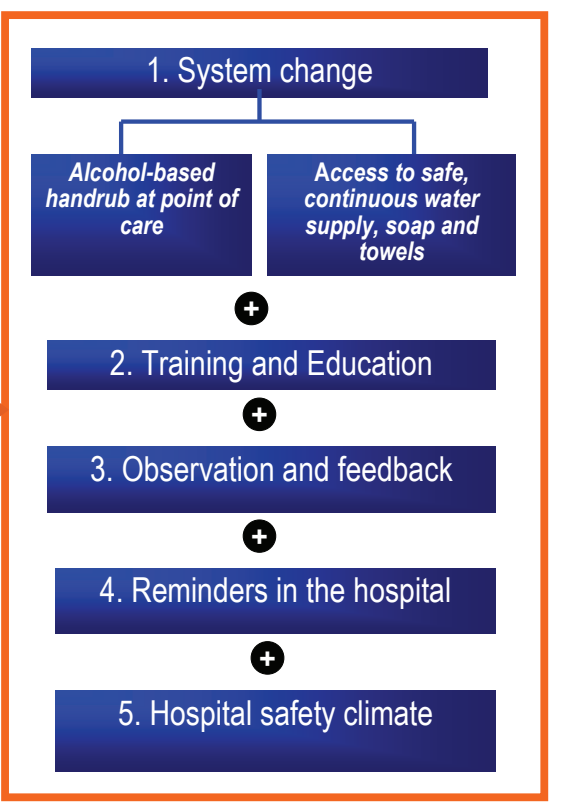

alcohol-based handrub via the commercial market is limited or unaffordable. For this reason, a number of the pilot sites are manufacturing alcohol-based hand rub according to the newly developed WHO formulation.

\section{Complementary Test Sites (CTS)}

As a result of the overwhelming number of requests from countries to be a pilot site, an unforeseen category was created in early 2007, i.e. Complementary Test Sites (CTS). Over 300 facilities are now participating in implementing and evaluating the Guideline strategy and participation is open to all health-care facilities around the world. On enrolment, CTSs have access to all of the implementation resources and can participate in a specially designed web-based community forum. The condition to become a CTS is dependent on the facility providing feedback to $\mathrm{WHO}$ on feasibility and appropriateness of tools. Support offered by the WHO WAPS to Complementary Test Sites is limited.

Table 1: The Five Moments Concept

- Reaching a consensus on the levels of risk relating to different care activities has proved difficult throughout the history of hand hygiene improvement ;

- The Five Moments concept defines key moments for hand hygiene action - the why, when and how to apply hand hygiene during routine care activity;

- The aim of the Five Moments is a unified vision and a strong sense of ownership.

- Aligns with evidence-base concerning the spread of HAI and multi resistant organisms;

- Presents an ergonomic fit: interwoven within the natural workflow of care

- Easy to learn;

- Logical;

- Applicable in a wide range of settings. 

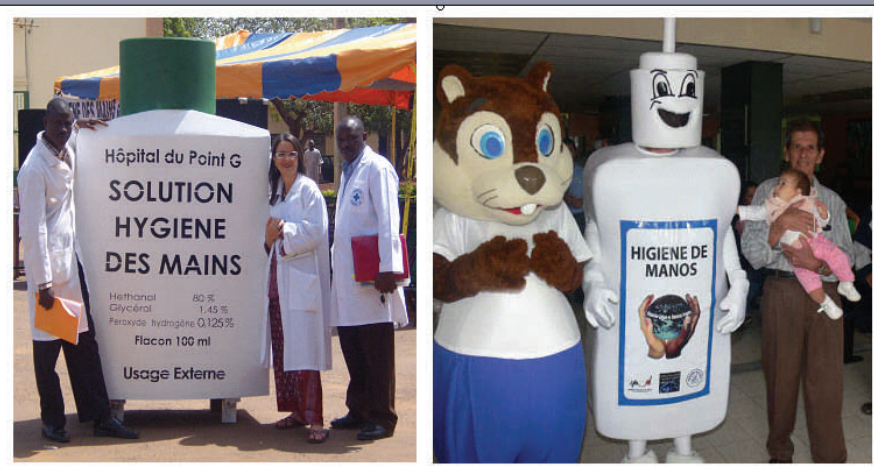

Figure 3: Pilot site launch of Step 3 (active phase) of the strategy: Mali (left) and Costa Rica (right)

\section{Country Commitment and campaigning}

In a little over two years, eighty-two countries/ autonomous regions have made a national commitment to tackle HAI. This represents about $74 \%$ of the world population (Figure 4, status at December 2007).

There has also been growing support for national campaigning, particularly in countries which have made a national commitment. We are aware of at least 22 countries initiating or strengthening national hand hygiene improvement campaigns. This presents significant opportunities for information exchange both between and beyond the existing campaigners. The Alliance is particularly interested in how the campaign approach to hand hygiene improvement can spread to low and middle income countries.

Since the launch of the First Global Patient Safety Challenge in 2005 considerable progress has been made to raise awareness of the importance of HAI as a patient safety problem. More recently, the WHO WAPS and IFIC have joined forces to invite all of IFICs specialist professional organizations to demonstrate commitment to the Challenge and take action in their respective countries. In reality this could mean each member organization signing a statement of commitment to Clean Care is Safer Care.

\section{8 and beyond}

The next phase of the Challenge is focused on completing the pilot and complementary testing and evaluation. Learning from this phase is critical in order to ensure a final version of the Guidelines and all related tools is published in the autumn of 2008. In parallel, work to address the unresolved issues identified during the initial guideline preparation, relating to water quality, alcoholbased handrubs and patient involvement, will continue during 2008. Patient involvement in hand hygiene improvement and the prevention of HAI is paramount, and the perceptions and experiences of patients on this subject are currently being sought via a web-based survey targeting all WHO regions.

\section{Conclusion:}

The First Global Patient Safety Challenge is concerned with improving the safety of patients through better infection control. Good hand hygiene practice is central to a clean safe approach to patient care and in many countries hand hygiene improvement is being embraced at the highest level. It provides a natural opening into other areas of infection control and safety and is relevant in low to middle income, as well as high income countries.

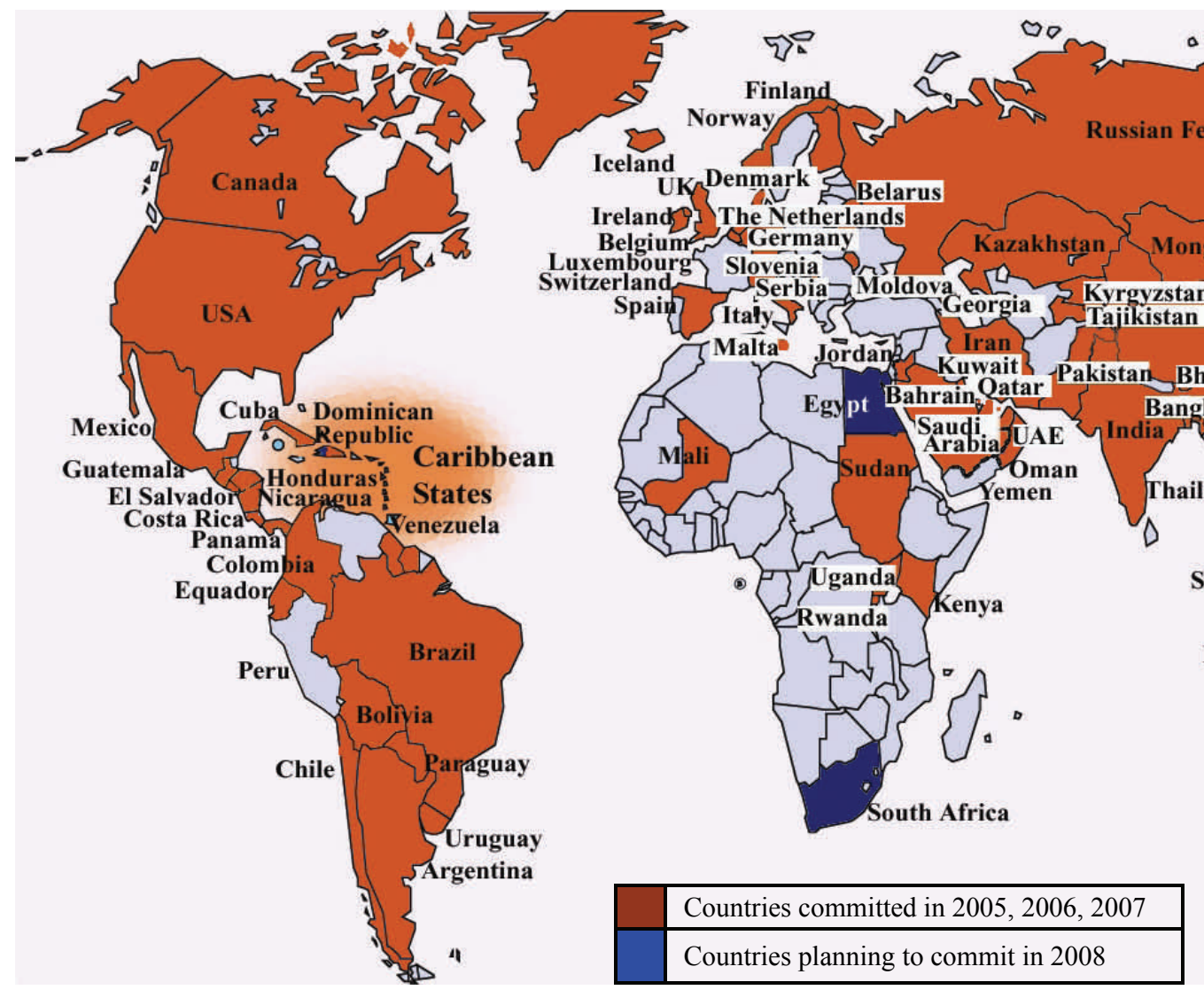

Figure 4: The 82 countries/SARs pledging to tackle HAI
Countries planning to commit in 2008
Countries planning to commit in 2008
Page 3 not for citation purposes 
Acknowledgements:

The authors are indebted to the group of international experts and WHO members who worked on the development of the Global Patient Safety Challenge, in particular for their participation to the two international WHO Consultations, review of the available scientific evidence, writing of the WHO draft Guidelines on Hand Hygiene in Health Care, and fostering discussion among authors and members of the different Task Forces and working groups. The complete list of participants in the development of the Guidelines documents is available at http://www.who.int/gpsc/ events/2005/en/index.html (accessed December 14th 2007) The authors also express their gratitude to the Patient Safety team and other WHO staff from all the departments involved at Headquarters and in the Regional and Country Offices for their work.

Didier Pittet also wishes to thank the members of the Infection Control Programme at the University of Geneva Hospitals, and Rosemary Sudan for providing editorial assistance and outstanding support.

\section{References}

1. The WHO Guidelines on Hand Hygiene in Health Care, Advanced Draft (2006) WHO

2. Sax H, Allegranzi B, Uckay I, Larson E, Boyce J, Pittet D (2007) 'My five moments for hand hygiene': a user-centred design approach to understand, train, monitor and report hand hygiene. Journal of Hospital Infection (2007) 67, 9-21

\section{Useful sources of information}

Website of the World Alliance for Patient Safety http://www.who.int/patientsafety/en/

Safe blood, injection and immunization, water and sanitation and emergency and surgical procedures

http://www.who.int/gpsc/elements/en/index.html

How to enroll as a Complementary Test Site

http://www.who.int/gpsc/country work/pilot testing info/en/ index.html

National Campaigns Report

http://www.who.int/patientsafety/events/07/

ps_meeting_report_geneva_29august.pdf

The Patient Involvement Report (Phase 1)

http://www.who.int/patientsafety/

hand hygiene survey phase1.pdf

The Patient Involvement Survey

http://www.who.int/gpsc/patient survey/en/index.html

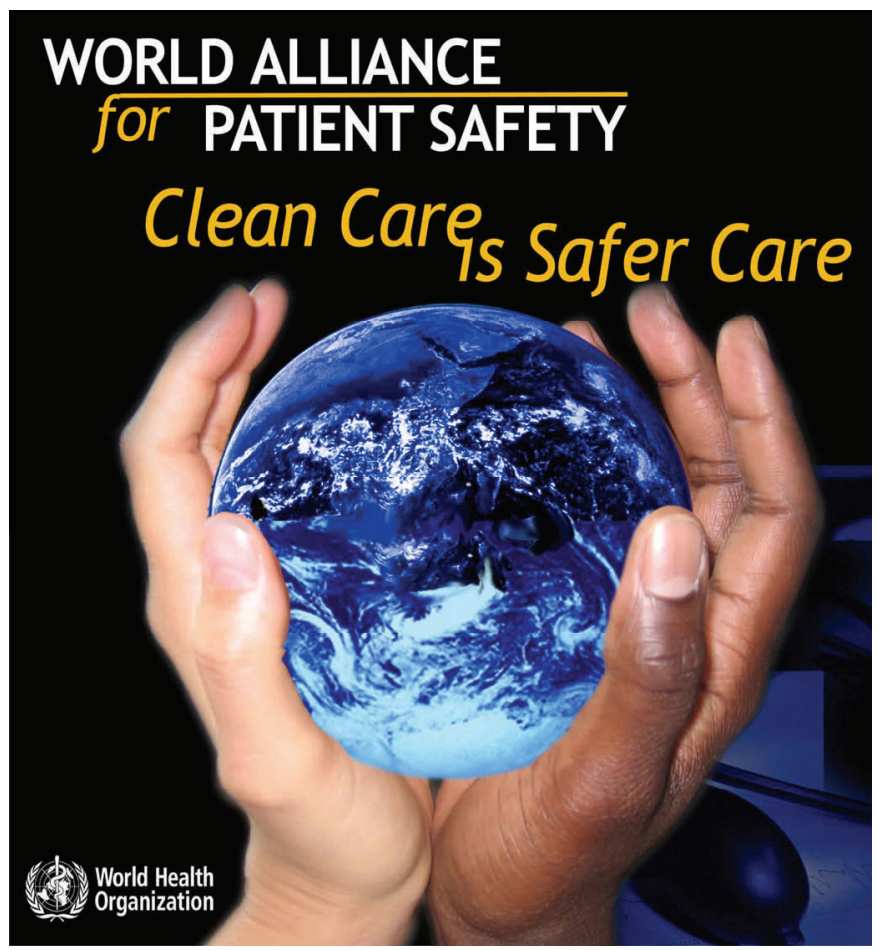

